\title{
Energy-sparing adaptations in human pregnancy assessed by whole-body calorimetry
}

\author{
BY A. M. PRENTICE, G. R. GOLDbERG, H. L. DAVIES, P. R. MURGATROYD \\ AND W. SCOTT \\ MRC Dunn Nutrition Unit, Milton Road, Cambridge, CB4 IXJ
}

(Received 11 August 1988 - Accepted 21 February 1989)

\begin{abstract}
The hypothesis that the energy cost of human pregnancy can be minimized by energy-sparing metabolic adaptations was tested using serial $24 \mathrm{~h}$ whole-body calorimetry. Eight healthy, well-nourished women were studied prepregnant and at $6,12,18,24,30$ and 36 weeks gestation. Basal metabolic rate (BMR) showed highly characteristic changes within each subject and large inter-individual differences $(F 3.5, P$ $<0.01$ ). Some subjects showed a highly significant depression of metabolism up to 24 weeks gestation in support of the initial hypothesis. At 36 weeks BMR ranged from +8.6 to $+35.4 \%$ relative to the prepregnant baseline. This wide variability was not explained by differences in the amount of lean tissue gained. Women displaying the energy-sparing suppression of BMR tended to be thin, suggesting that changes in metabolism may be responsive to initial energy status ( $\triangle$ BMR $v$. prepregnant body fat: $r$ $0.84, P<0.005)$. Changes in $24 \mathrm{~h}$ energy expenditure closely paralleled changes in BMR $(r 0.98, P<$ 0.001 ), since the energy cost of minor voluntary activity and thermogenesis remained very constant within each individual. Pregnancy decreased the net cost of weight-dependent and weight-independent standard exercises when expressed per $\mathrm{kg}$ body-weight: stepping -10 (SD 2) \%, $P<0.001$ at 18-36 weeks, cycling -26 (so 7$) \%, P<0.01$ at $12-36$ weeks. The average integrated maintenance costs of pregnancy matched previous group estimates from well-nourished women, but individual estimates ranged from -16 to $+276 \mathrm{MJ}$ (coefficient of variation $93 \%$ ). This high level of variability has important implications for the prescription of incremental energy intakes during pregnancy. It may also have had evolutionary significance.
\end{abstract}

Energy-sparing adaptations: Pregnancy: Whole-body calorimetry.

Our previous studies on the energy cost of human pregnancy in under-nourished Gambian women have suggested that important metabolic adaptations can spare significant amounts of energy and thus protect fetal growth (Lawrence et al. 1987; Prentice \& Whitehead, 1987). Comparative studies in better nourished women show no such adaptations in grouped values (Forsum et al. 1985; Durnin et al. 1987; Throngprasert et al. 1987; Tuazon et al. 1987; van Raaij et al. 1987). However, the use of cross-sectional analysis in these studies may have obscured the presence of adaptive responses within certain individuals.

The present study was designed to investigate inter-subject variability in the metabolic responses to pregnancy by serial analysis of prospectively-collected data from a prepregnant baseline. It addressed the specific hypothesis that in certain women a reduction in metabolism could be of sufficient magnitude to spare the entire maintenance costs of the fetus and other products of conception. 
A. M. PRENTICE AND OTHERS

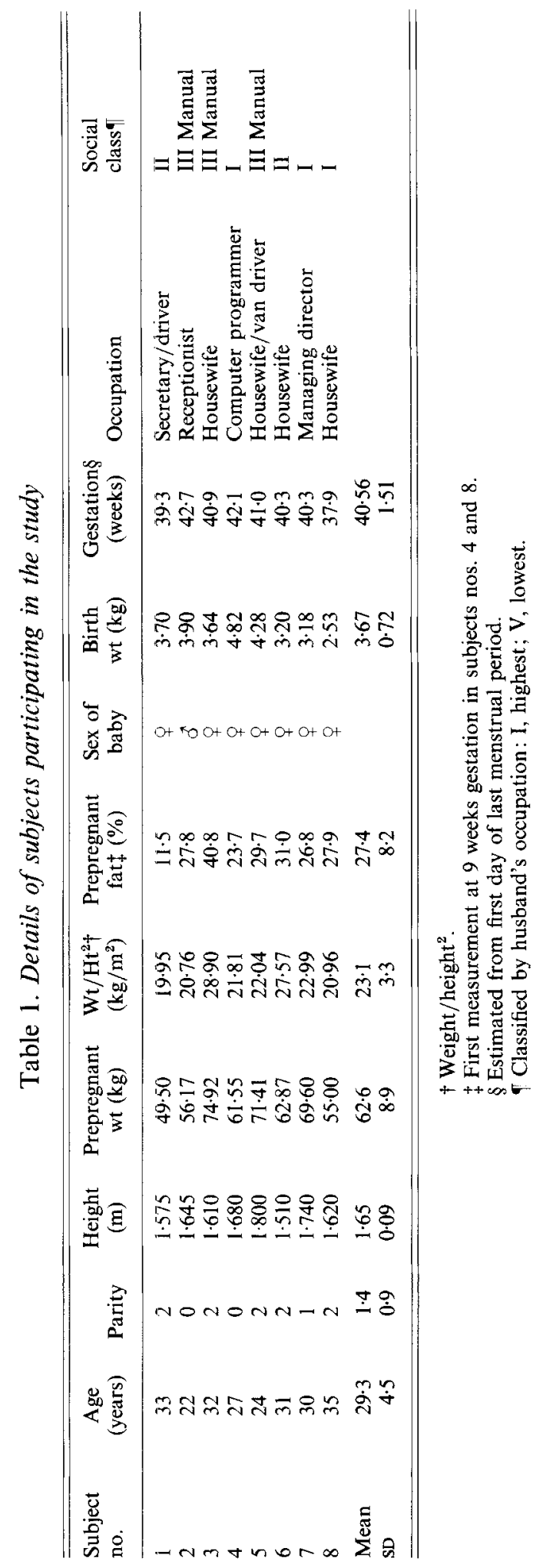




\section{SUBJECTS AND METHODS}

\section{Subjects}

Sixty women responded to advertisements seeking prepregnant volunteers and eighteen agreed to participate in the study after a detailed explanation of all the procedures involved. All subjects were healthy but no other selection criteria were used. Two of the participants were found to be pregnant already, two dropped out of the study, five failed to conceive and one miscarried. Details of the eight women who completed the study are given in Table 1. The group was broadly representative of the general population. Two subjects were grade 1 obese according to the weight $/$ height $^{2}$ classification (body mass index). Subject no. 1 was a smoker and subject no. 6 an ex-smoker.

The study was approved by the Dunn Nutrition Unit and Cambridgeshire Area Health Authority Ethical Committees.

\section{Study design}

Baseline calorimetry measurements in the non-pregnant, non-lactating (NPNL) state were made in the luteal phase of the menstrual cycle between days 20 and 25 from the first day of the previous menses. Subjects nos. 1, 4, 6 and 8 had, in fact, conceived at ovulation and were therefore between 7 and $14 \mathrm{~d}$ post-conception at the time of the baseline calorimetry, but will be classified as NPNL.

Following conception, measurements were made at 6-weekly intervals until 36 weeks gestation. Subject no. 2 missed the 6 week measurement due to a threatened miscarriage and subject no. 7 due to the loss of a twin fetus. Interpolated values have been used when calculating group averages. Subject no. 8 missed the 36 week measurement due to hospitalization for pre-eclampsia.

Whole-body calorimetry

Energy expenditure was measured in the Dunn Clinical Nutrition Centre's pair of $11 \mathrm{~m}^{3}$ open-circuit indirect calorimeters. The chambers were ventilated with fresh air at 200 litres/min. Air within the calorimeters was recirculated via a conditioning unit at 20000 litres/min to ensure rapid mixing and even temperature control at $26 \pm 0 \cdot 2^{\circ}$. Ventilation rate was controlled on the inlet side by Rotameter flow meters (KDG Flowmeters Ltd, Burgess Hill, Sussex) calibrated against a wet gas meter. Humidity was not controlled and, therefore, fluctuated according to ambient humidity, but remained slightly higher due to the subject's expiratory and insensible water losses.

The calorimeters were manually calibrated at the beginning of each run using pure nitrogen to set analyser zeros, and accurately-assayed carbon dioxide in air (nominally $1 \%$ ) and fresh air to set full-span readings. Subsequent gas sampling and analysis, data acquisition and storage were controlled by a Hewlett-Packard 1000 Series mini-computer interfaced via a Solartron 3510 Integrated Measurement System (Solartron Electronic Group Ltd, Farnborough, Hampshire). Gas samples were analysed every $200 \mathrm{~s}$ using a repeating cycle of five calorimeter measurements followed by one fresh air measurement. Every $2 \mathrm{~h}$ the gas analyser zero and full-span readings were automatically checked against reference gases as described previously. Corrections for meter drift were applied by assuming linear trends. During these calibration check-routines, calorimeter measurements were interpolated. The protocol was designed to ensure that critical measurement periods (e.g. basal metabolic rate (BMR) or exercise) never coincided with meter calibration. Barometric pressure (BHL-4100 Absolute Pressure Transducer; Bell \& Howell Ltd, Basingstoke, Hampshire), dew point (DP-1100 AP Condensation Dew Point Hygrometer; General Eastern Instrument Corporation, Watertown, Massachusetts) and temperature 
(laboratory-built thermocouples) of the calorimeter and fresh air samples were measured before gas analysis using Model 0A 184 paramagnetic oxygen analysers (Servomex Ltd, Crowborough, Sussex) and Model IRGA 40 infra-red $\mathrm{CO}_{2}$ analysers (J. \& S. Sieger Ltd, Poole, Dorset) (later replaced by Model 12X1, Servomex Ltd, Crowborough, Sussex). The $\mathrm{O}_{2}$ analysers were used in the single-channel mode. Accuracy of the $\mathrm{CO}_{2}$ analysers was improved by applying computer-fitted calibration curves obtained by gas dilution.

Energy expenditure was calculated from Weir's (1949) equation using rapid-response calculations described in detail elsewhere (Brown et al. 1984). These calculations produce a $95 \%$ response time of $400 \mathrm{~s}$ in our calorimeters. Precision and accuracy of the calorimeters were periodically checked using controlled infusions of pure $\mathrm{N}_{2}$ and $\mathrm{CO}_{2}$ to simulate a subject. Typical results showed instrumentation noise to be approximately $\pm 0.09, \pm 0.04$ and $\pm 0.008 \mathrm{~kJ} / \mathrm{min}$ over $0.5,1$ and $24 \mathrm{~h}$ periods respectively.

The calorimeters were furnished with a folding bed, comfortable chair, table, bicycle ergometer, stepping block, television, radio-cassette player, telephone, intercom and toilet facilities. Food and drink were passed into the chamber, and faecal and urine samples taken out of the chamber via separate air locks. Subjects were under continual supervision by the experimenters or night nurses. Large windows provided contact with the outside. All subjects enjoyed being in the chambers and can be assumed to have been unstressed by the procedure.

\section{Calorimeter protocol}

Subjects nos. 1 and 6 only stayed in the calorimeter for overnight and BMR measurements. The other six subjects followed a $36 \mathrm{~h}$ protocol in which all activity was strictly controlled according to a set timetable which remained the same for all subjects and throughout pregnancy. The protocol timing was as follows: 18.00-19.00 hours medical examination and supper; 20.00 hours, enter calorimeter; 22.30 hours prepare for bed (wash, undress, rearrange furniture); 23.00 hours lights out; 08.00 hours woken for $60 \mathrm{~min}$ BMR measurement ; 09.00 hours get up (wash, dress, rearrange furniture); 09.30 hours breakfast; 12.30 hours weight-independent exercise for $30 \mathrm{~min}$ (cycling at 25 watts); 13.30 hours lunch; 16.30 hours weight-dependent exercise for 30 min (block-stepping at ten steps per $\min \times 220 \mathrm{~mm}$ ); 19.00 hours supper; then as for night 1 through to the end of a second BMR measurement. Subjects had periods of obligatory standing as follows: $2 \times 30 \mathrm{~min}$ dressing and undressing; $5 \mathrm{~min}$ before each exercise; $10 \mathrm{~min}$ after each exercise; $2-3$ min collecting lunch and supper from hatch; total $=100 \mathrm{~min}$. Except where indicated otherwise, subjects remained seated and were only permitted very light activities such as watching television, reading, knitting, sewing, writing and eating. The purpose of using such a rigid protocol was to eliminate behavioural noise which may have obscured the underlying physiological changes. Under such conditions the within-subject repeatability of $24 \mathrm{~h}$ energy expenditure is approximately $1.6 \%$ (Dallosso et al. 1982).

BMR was measured immediately on waking, $13 \mathrm{~h}$ post-absorptive, at thermoneutrality and at complete physical rest, thus satisfying the classically defined conditions. The coefficient of variation (CV) for repeat measurements was calculated by comparing thirtyeight pairs of BMR measurements on consecutive mornings in the six subjects who underwent $36 \mathrm{~h}$ calorimetry. The mean difference between BMR 1 and 2 was $-0.87 \%$ with a $\mathrm{CV}$ of $2.6 \%$ (indicating no significant order effect). This precision improves to $1.9 \%$ since values are presented as the mean of duplicate BMR.

Exercise rates were controlled by electronic metronome and were closely supervised. In another study the CV for repeat 30 -min measurements were $3.0 \%$ for cycling and $4.3 \%$ for stepping.

The total energy cost of activity plus thermogenesis (AT) was calculated by subtracting BMR from the $24 \mathrm{~h}$ energy expenditure measured from 09.00 to 09.00 hours. The energy 
cost of minor physical movements plus thermogenesis was then computed by further subtracting the energy expended during the two exercise periods and the dressing and undressing periods from AT.

Subjects were asked to avoid substantial changes to their usual diet on the day preceding calorimetry. The diet in the calorimeter was designed to maintain subjects in very slight negative energy balance. In eight of the thirty-eight measurements, expenditure exceeded intake by $0.5-1.0 \mathrm{MJ}$ and on two occasions the excess was $1.0-1.5 \mathrm{MJ}$. All other balances were within $0.5 \mathrm{MJ}$. The diet consisted of normal food with a protein-fat-carbohydrate mix of $14: 40: 46$ calculated by energy. Each meal provided exactly one-third of the day's intake.

\section{Body composition analysis}

Body-weight was measured immediately after each calorimetry run, after voiding, and corrected for the weight of clothing. Subjects had been fasting for $14 \mathrm{~h}$. A final measurement was made at 38 weeks gestation. Lean body mass (LBM) was calculated by deuterium dilution following oral dosing with $0.05 \mathrm{~g}^{2} \mathrm{H}_{2} \mathrm{O} / \mathrm{kg}$. Enrichment levels were measured by isotope-ratio mass spectrometry (VG Isogas, Middlewich, Cheshire) and dilution spaces were calculated by back extrapolation to time zero of serial daily urine samples. In the NPNL state LBM was assumed to be $73 \%$ water. At subsequent time points average values from the literature were assumed for the water content of (a) amniotic fluid, expanded plasma volume and extracellular fluid, and (b) the excess hydration (above $73 \%$ ) of the remaining products of conception. These were converted to a per $\mathrm{kg}$ basis and applied pro rata according to the subject's current weight. The corrections at each 6-weekly interval were: (a) $1 \cdot 5,4 \cdot 5,11 \cdot 0,22 \cdot 0,28 \cdot 0,34 \cdot 0 ;$ (b) $0,0 \cdot 5,1 \cdot 5,2 \cdot 5,3 \cdot 0,3 \cdot 0 \mathrm{~g}$ water $/ \mathrm{kg}$. This approach can only be considered to be a best estimate and does not account for possible oedema. However, reverse calculation of the apparent water content of lean tissue yielded a value of 76.8 (SD 0.5$) \%$ at 36 weeks gestation. This agrees closely with the value of 76.6 (SD 3.2) \% obtained by simultaneous ${ }^{40} \mathrm{~K}$ and total body water estimates in women close to term published elsewhere (Seitchik, 1967). The first LBM measurement in subjects nos. 4 and 8 was made 9 weeks after the first day of the last menstrual period. Baseline LBM has been calculated by subtracting the mean gain of $0.5 \mathrm{~kg}$ measured in the other subjects.

\section{RESULTS}

The average weight gain up until the final measurement at 38 weeks gestation was 14.4 (SD $4 \cdot 1) \mathrm{kg}$ with a range from 8.5 to $21 \cdot 1 \mathrm{~kg}$ (Fig. 1). When expressed as percentage increments above the NPNL weight there was still considerable variation between subjects with total weight gains varying between 13.8 and $30.7 \%$. There was no detectable relation between percentage weight gain and initial weight or body mass index.

LBM showed a smooth increase when averaged for the whole group (Fig. 2). The mean increment at 36 weeks was 6.7 (SD 1.6) kg. Fig. 2(b) illustrates that the changes in LBM were remarkably consistent between individuals in spite of the wide range in initial body size and showed smooth changes in most cases. The apparent small losses of LBM during early pregnancy in subjects nos. 5 and 6 and during mid-pregnancy in subjects nos. 1 and 7 may partly reflect measurement noise since the precision of LBM estimates based on ${ }^{2} \mathrm{H}_{2} \mathrm{O}$ dilution is about $\pm 1 \%$ which is equivalent to $\pm 500 \mathrm{~g}$ in these subjects. However, subject no. 7 did lose a twin fetus in early pregnancy and the plateau in LBM may, therefore, have been real.

The increase in LBM was also calculated as a percentage of the initial value, since it is reasonable to assume that the size of the products of conception would depend on a woman's non-pregnant stature. When expressed in this way the changes in LBM were 


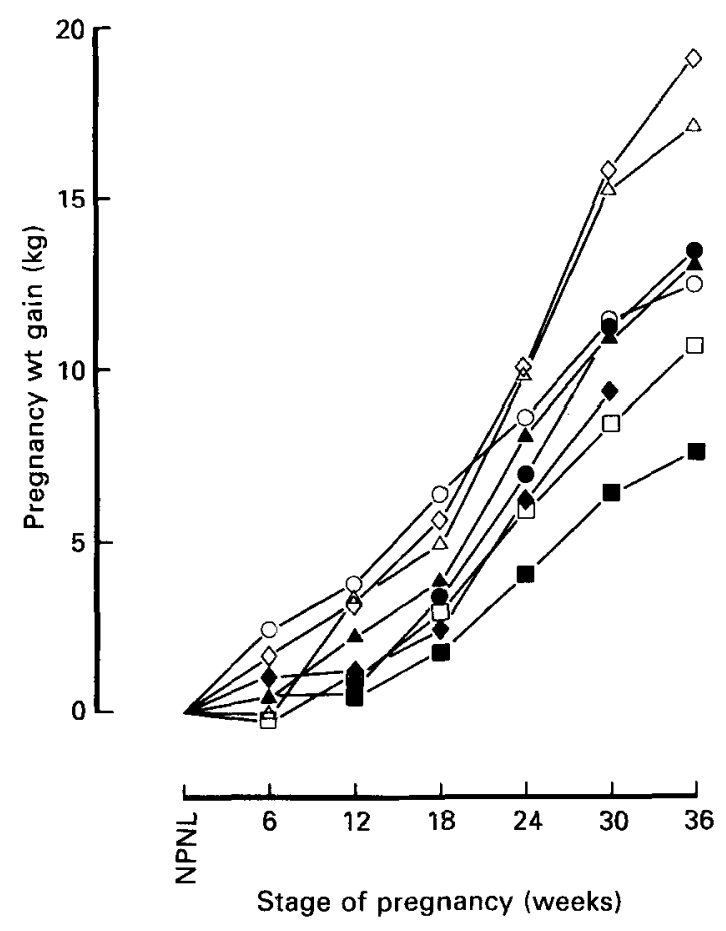

Fig. 1. Increase in body-weight during pregnancy for eight healthy, well-nourished women. Subject nos: $1(\mathrm{O})$, $2(\bigcirc), 3(\square), 4(\square), 5(\Delta), 6(\Delta), 7(\diamond), 8(\bullet)$. NPNL, non-pregnant, non-lactating. For details of subjects see Table 1.

remarkably similar in all except subject no. 2 who showed a higher incremental gain. There was no clinical evidence of oedema in this subject. Subjects nos. 7 and 8 showed an unusually raised LBM at the final measurement which can be ascribed to clinically apparent oedema in both cases.

Fig. 3 shows the changes in BMR expressed on a whole-body basis and as a percentage of the NPNL value. The group average showed no change up to 18 weeks gestation followed by a steep increase to reach +20.0 (sD 10.1$) \%$ at 36 weeks. Fig. $3(b)$ shows that the high standard deviation on the group mean arises not from measurement noise, but from very significant inter-individual differences in the response to pregnancy. With the exception of some minor early deviations, all women showed very smooth and characteristic BMR curves. (Analysis of variance: $F 3.50$; df 14,$28 ; P<0.01$ in linear and quadratic terms of fitted curves.) Individual BMR values are given in Table 2.

Subjects nos. 1 and 4 showed a highly significant depression of metabolism up until 24 weeks gestation, and even at 30 weeks their BMR was no higher than in the NPNL state. In contrast, subjects nos. 3, 5 and 7 showed an immediate and progressive rise in BMR. The remaining subjects showed intermediate changes, with a slight reduction in BMR up until 18 weeks gestation, followed by the steep increase which occurred in all women towards term. At 36 weeks the increment in BMR above the NPNL baseline varied between 8.6 and $35.4 \%$.

The total energy cost of changes in maintenance requirements during pregnancy can be calculated as the cumulative difference between pregnancy BMR (with extrapolation to 40 weeks) and the NPNL value. The average value for the group was 123 (SD 114) MJ. Due to the early suppression of metabolism in the energy-sparing subjects (nos. 1 and 4) the 


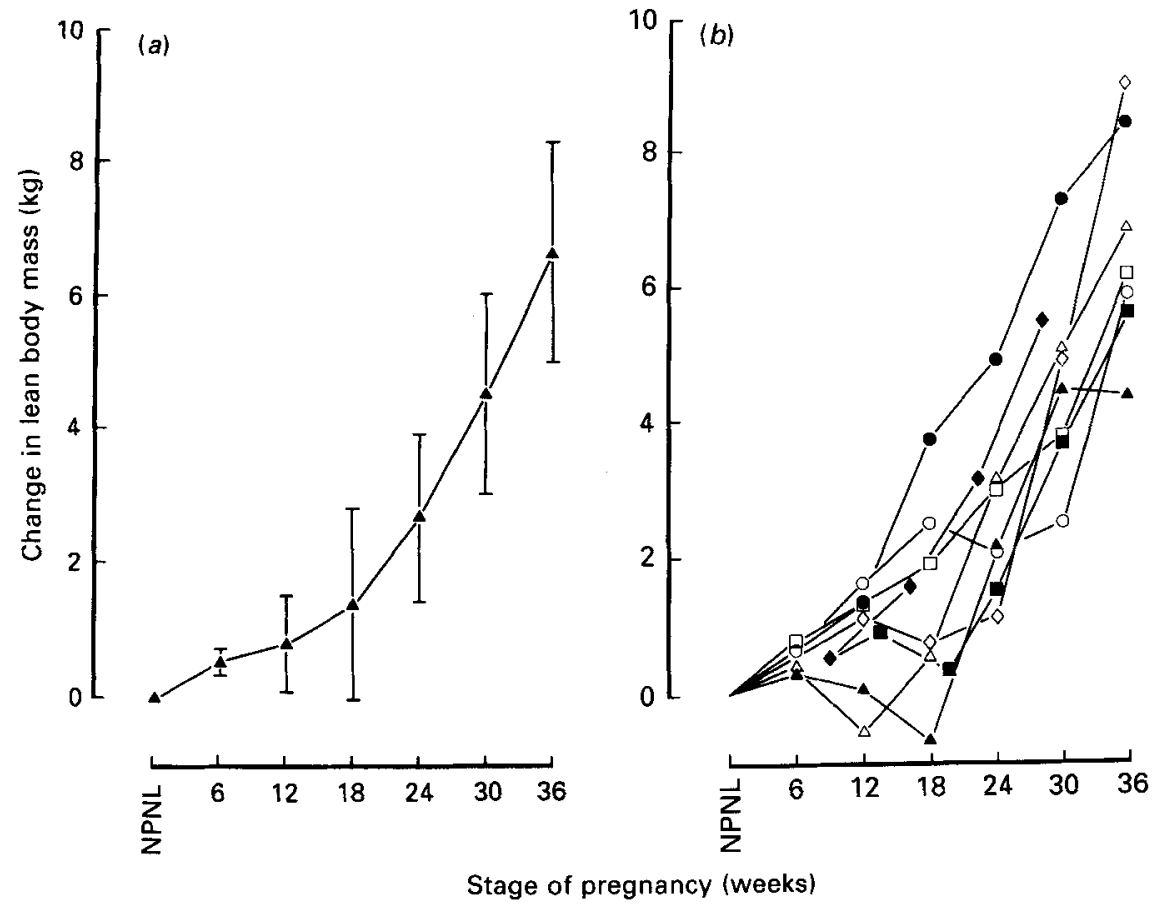

Fig. 2. Changes in lean body mass during pregnancy for eight healthy, well-nourished women. Subject nos: $1(O)$, $2(\bigcirc), 3(\square), 4(\square), 5(\Delta), 6(\Delta), 7(\diamond), 8(\diamond)$. (a) Group means and standard deviations represented by vertical bars, $(b)$ individual values. NPNL, non-pregnant, non-lactating.
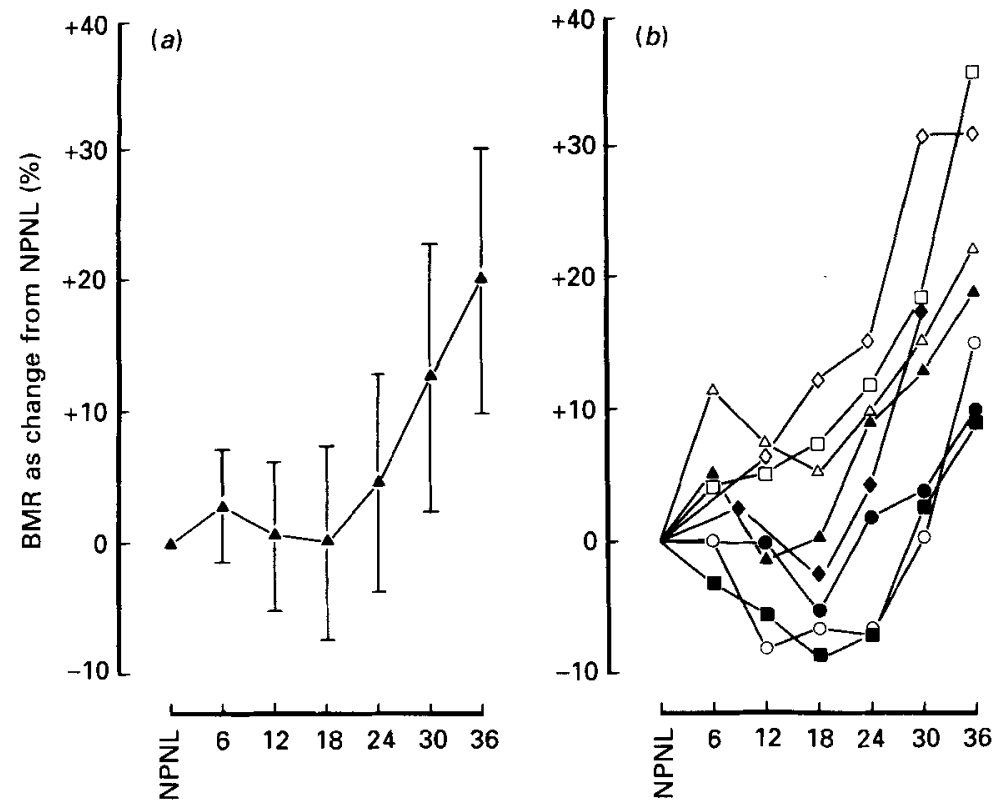

Stage of pregnancy (weeks)

Fig. 3. Changes in basal metabolic rate (BMR) during pregnancy for eight healthy, well-nourished women. Subject nos: $1(O), 2(\bigcirc), 3(\square), 4(\mathbf{O}), 5(\triangle), 6(\Delta), 7(\diamond), 8(\diamond)$. (a) Group means and standard deviations represented by vertical bars, $(b)$ individual values. Average non-pregnant, non-lactating (NPNL) BMR was 6113 (SD 356 ) kJ/d. 
Table 2. Individual basal metabolic rate values $(k J / d)$

(Values are means with standard errors calculated from the fifteen $200 \mathrm{~s}$ measurements within each hour. Values in parentheses are $\mathrm{kJ} / \mathrm{d}$ per $\mathrm{kg}$ lean body mass)

\begin{tabular}{|c|c|c|c|c|c|c|c|c|c|c|c|c|c|}
\hline \multirow[b]{3}{*}{ Subject no. } & \multicolumn{13}{|c|}{ Stage of pregnancy (weeks) } \\
\hline & \multicolumn{2}{|c|}{ NPNL } & \multicolumn{2}{|l|}{6} & \multicolumn{2}{|l|}{12} & \multicolumn{2}{|l|}{18} & \multicolumn{2}{|l|}{24} & \multicolumn{2}{|l|}{30} & 36 \\
\hline & Mean & $\mathrm{SE}$ & Mean & $\mathrm{SE}$ & Mean & $\mathrm{SE}$ & Mean & $\mathrm{SE}$ & Mean & SE & Mean & $\mathrm{SE}$ & Mean \\
\hline 1 & \multicolumn{2}{|c|}{$(142)$} & \multicolumn{2}{|c|}{$\begin{array}{c}61800_{(140)}^{73} \\
\end{array}$} & \multicolumn{2}{|c|}{$(126)$} & \multicolumn{2}{|c|}{$(125)$} & \multicolumn{2}{|c|}{$(126)$} & \multicolumn{2}{|c|}{$(134)$} & (143) \\
\hline 2 & \multicolumn{2}{|c|}{$(144)$} & \multicolumn{2}{|c|}{ - } & \multicolumn{2}{|c|}{ (138) } & \multicolumn{2}{|c|}{$(125)$} & \multicolumn{2}{|c|}{$(130)$} & \multicolumn{2}{|c|}{$(127)$} & $\begin{array}{c}6415 \quad 61 \\
(133)\end{array}$ \\
\hline 3 & \multicolumn{2}{|c|}{$(151)$} & \multicolumn{2}{|c|}{ (154) } & \multicolumn{2}{|c|}{$(154)$} & \multicolumn{2}{|c|}{$(155)$} & \multicolumn{2}{|c|}{$(158)$} & \multicolumn{2}{|c|}{$(164)$} & (179) \\
\hline 4 & \multicolumn{2}{|c|}{ (136) } & \multicolumn{2}{|c|}{$(130)$} & \multicolumn{2}{|c|}{$(126)$} & \multicolumn{2}{|c|}{ (123) } & \multicolumn{2}{|c|}{$(122)$} & \multicolumn{2}{|c|}{$(129)$} & $(132)$ \\
\hline 5 & $\begin{array}{r}6385 \\
(1\end{array}$ & $\begin{array}{l}112 \\
27)\end{array}$ & 7110 & $\begin{array}{l}119 \\
11)\end{array}$ & 6845 & & 6710 & $2)^{75}$ & $\begin{array}{r}7005 \\
(1\end{array}$ & 88 & 7350 & 3) & $\begin{array}{c}7795 \quad 186 \\
(136)\end{array}$ \\
\hline 6 & 5900 & $\begin{array}{l}175 \\
6)\end{array}$ & 6200 & $\begin{array}{l}221 \\
12)\end{array}$ & 5810 & $\begin{array}{l}110 \\
4)\end{array}$ & $\begin{array}{r}5920 \\
(1\end{array}$ & $\begin{array}{l}117 \\
9)\end{array}$ & $\begin{array}{r}6430 \\
(1\end{array}$ & $\begin{array}{l}190 \\
2)\end{array}$ & 6650 & $\begin{array}{l}87 \\
0)\end{array}$ & $\begin{array}{c}7000 \quad 77 \\
(147)^{7}\end{array}$ \\
\hline 7 & 6050 & $\begin{array}{l}120 \\
18)\end{array}$ & & 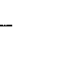 & 6440 & $\begin{array}{l}180 \\
4)\end{array}$ & ${ }^{6775}$ & 113 & $\begin{array}{l}6950 \\
(1\end{array}$ & 184 & 7915 & $\begin{array}{l}118 \\
1)\end{array}$ & $\begin{array}{c}7920 \quad 151 \\
(132)\end{array}$ \\
\hline 8 & ${ }^{5555}$ & 15) & 5685 & & & & 5405 & 66 & $\begin{array}{r}5790 \\
(1\end{array}$ & $10)^{79}$ & 6515 & $\begin{array}{l}93 \\
9)\end{array}$ & - \\
\hline
\end{tabular}

NPNL, non-pregnant, non-lactating,
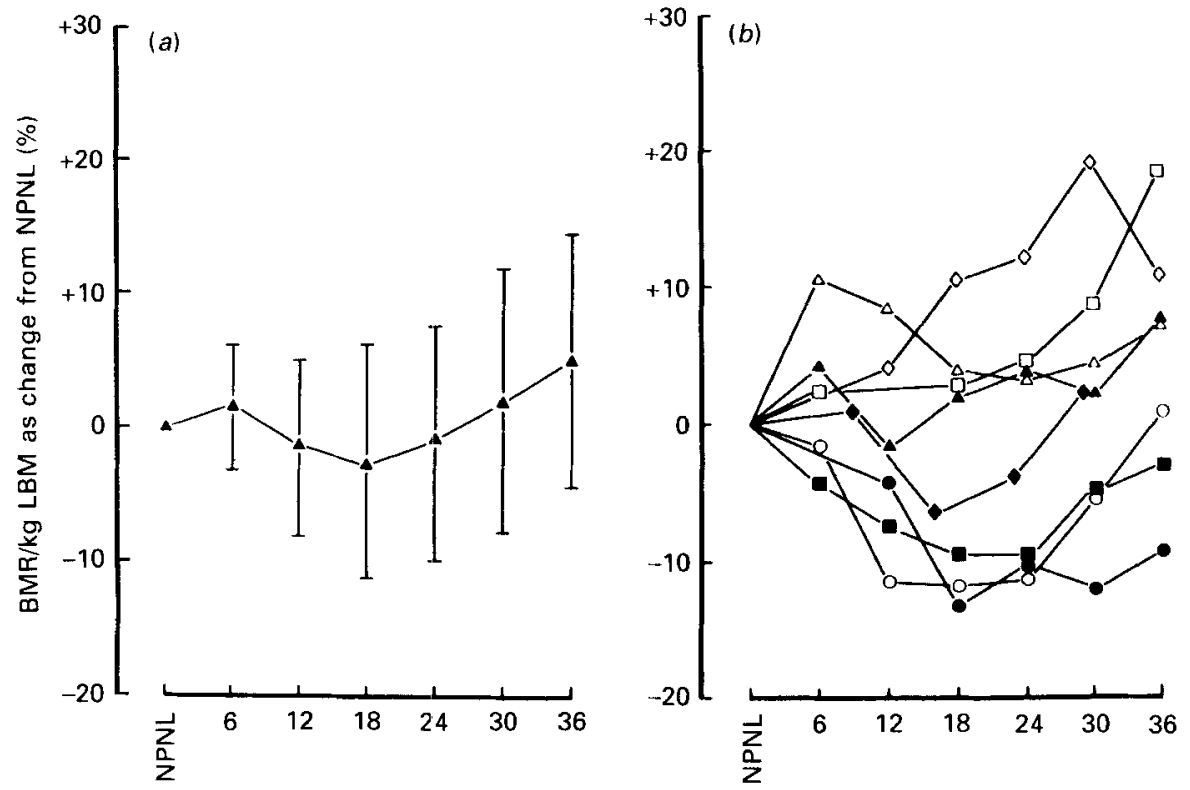

Stage of pregnancy (weeks)

Fig. 4. Mass-specific changes in basal metabolic rate (BMR) during pregnancy for eight healthy, well-nourished women. Subject nos: $1(\bigcirc), 2(\bullet), 3(\square), 4(\square), 5(\triangle), 6(\Delta), 7(\diamond), 8(\diamond)$. Values are expressed per $\mathrm{kg}$ lean body mass (LBM). (a) Group means and standard deviations represented by vertical bars, $(b)$ individual values. Average non-pregnant, non-lactating (NPNL) BMR was 137.1 (SD 10.8) kJ/kg LBM per d. 


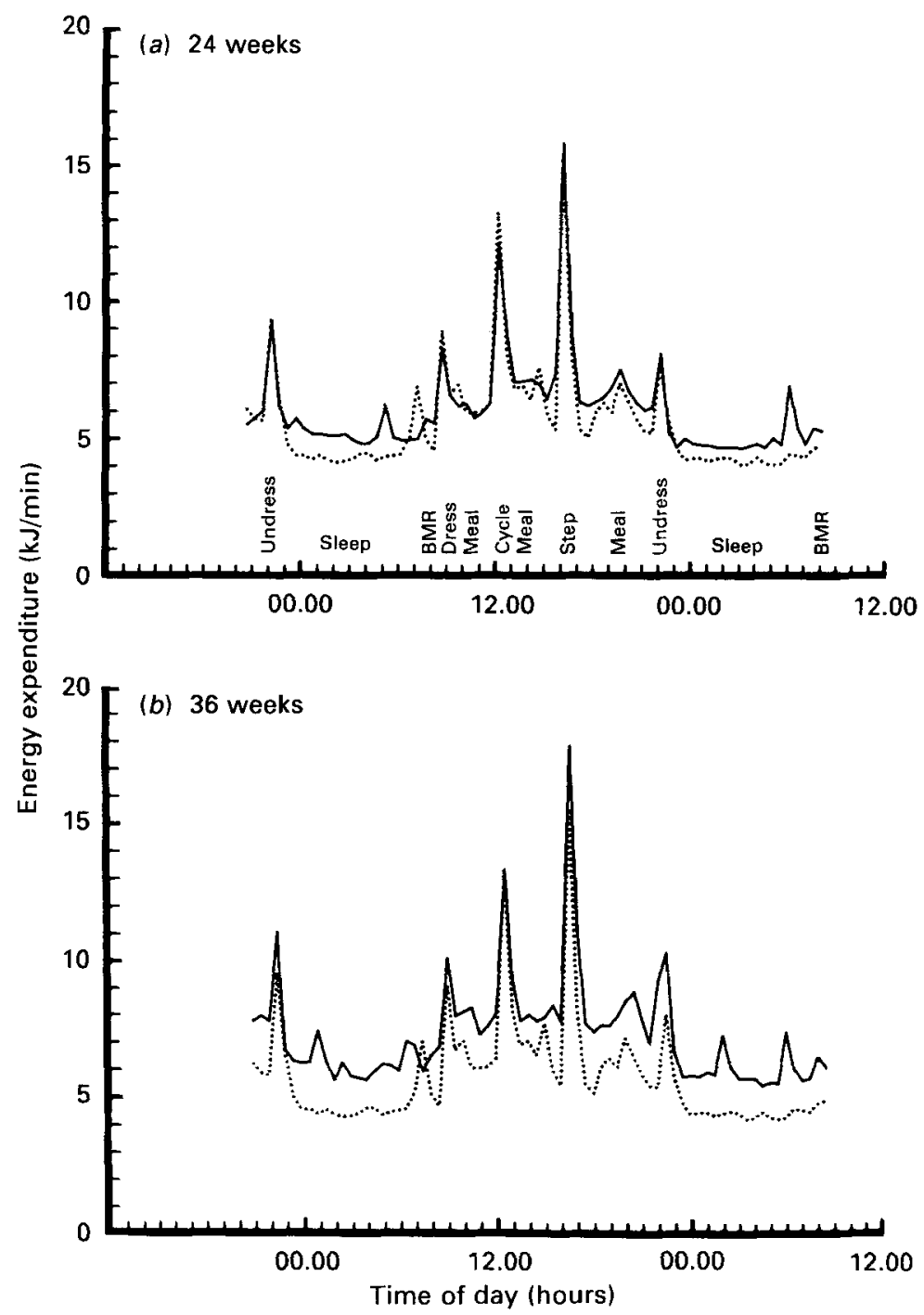

Fig. 5. Energy expenditure during $36 \mathrm{~h}$ whole-body calorimetry in an energy-profligate subject (subject no. 3). For details see Table 1. (--), Non-pregnant, non-lactating baseline; (-), expenditure at (a) 24 and (b) 36 weeks gestation. Values calculated over 30 min periods. BMR, basal metabolic rate.

integrated maintenance costs for the entire pregnancy were actually negative at -10 and $-16 \mathrm{MJ}$ respectively. In contrast, the maintenance costs for the two most energy-profligate subjects (nos. 3 and 7) were 276 and $266 \mathrm{MJ}$ respectively.

Fig. 4 shows the changes in BMR expressed per kg LBM. The group mean value showed no significant change throughout pregnancy. The slight indication of a rise towards term was not significant even when analysed by paired $t$ test within subjects. Fig. $4(b)$ clearly demonstrates that the differential metabolic responses illustrated in Fig. 3 were caused more by changes in mass-specific BMR than by differences in the amount of lean tissue gained. At term the change in $\mathrm{BMR} / \mathrm{kg} \mathrm{LBM}$ ranged from -9.2 to $+18 \cdot 6 \%$ relative to the NPNL value.

Figs. 5 and 6 illustrate the $36 \mathrm{~h}$ calorimeter traces from the most energy-profligate and 


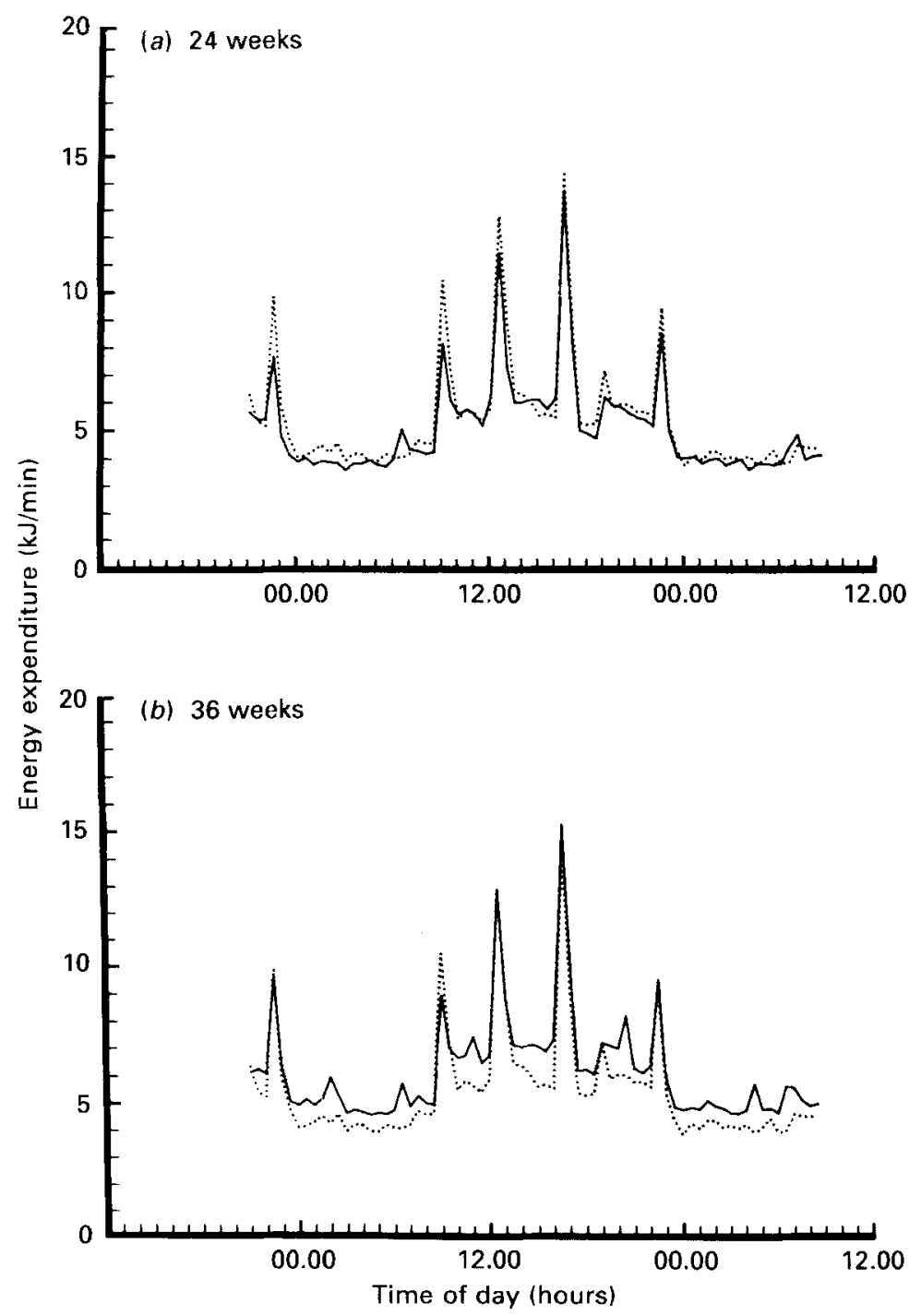

Fig. 6. Energy expenditure during $36 \mathrm{~h}$ calorimetry in an energy-sparing subject (subject no. 4). For details, see Table 1. (-.), Non-pregnant, non-lactating baseline; (-) expenditure at (a) 24 and $(b) 36$ weeks gestation. Values calculated over 30 min periods. BMR, basal metabolic rate.

most energy-sparing subjects respectively. In each case the 24- and 36-week measurements are superimposed on the NPNL baseline. The traces show results calculated by 30 -min intervals and plotted as point measurements for clarity. A more correct representation would be as a square-wave form for each $30 \mathrm{~min}$, but this makes it difficult to discriminate superimposed plots.

The relative energy costs of sleep, BMR, sitting quietly, diet-induced thermogenesis (after the evening meal) and of the various periods of activity are clearly illustrated. The small peaks of expenditure during some of the sleep periods occurred when subjects got up to pass urine. At 24 weeks gestation subject no. 3 expended considerably more energy than in the NPNL state for most of the day. In contrast, subject no. 4 expended significantly less energy for most of the day in spite of the fact that her body-weight and LBM had increased. 


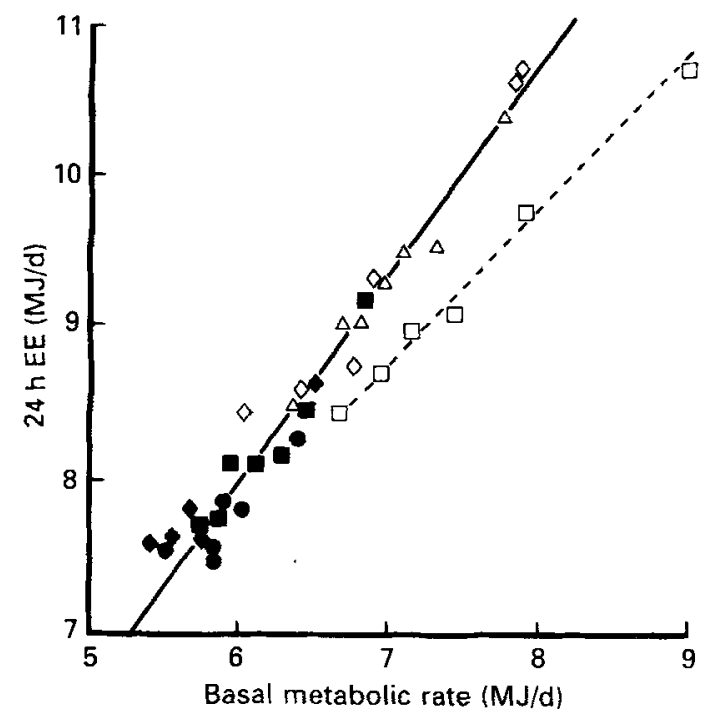

Fig. 7. Relation between basal metabolic rate (BMR) and $24 \mathrm{~h}$ energy expenditure (EE) in a whole-body calorimeter for six healthy, well-nourished women. Subject nos: $2(0), 3(\square), 4(\mathbf{0}), 5(\triangle), 7(\diamond), 8(\diamond) ; 24 \mathrm{~h}$ expenditure calculated from 09.00 hours on second day. Regression excluding subject no. 3 gives:

$$
24 \mathrm{~h} \mathrm{EE}=1.29 \mathrm{BMR}+284(\mathrm{~kJ} / \mathrm{d}) ; r 0.981 ; n 31 ; P<0.001 \text {. }
$$

Regression for subject no. 3 separately gives:

$$
24 \mathrm{~h} \mathrm{EE}=0.97 \mathrm{BMR}+1923(\mathrm{~kJ} / \mathrm{d}) ; r 0.994 ; n 6 ; P<0.001 \text {. }
$$

At 36 weeks gestation there was also a very marked difference between the substantially raised energy expenditure of subject no. 3 and the modest rise in subject no. 4 .

In five of the six subjects who participated in the full $36 \mathrm{~h}$ calorimetry there was a very close correlation between BMR and $24 \mathrm{~h}$ energy expenditure $(r 0.98, P<0.001)$ (Fig. 7 ). Each subject moved up or down the line of regression as her BMR changed. A close relation of this type would be anticipated given the facts that BMR represents a very high percentage of $24 \mathrm{~h}$ energy expenditure (averaging $75 \cdot 1 \%$ in these subjects) and that the subjects had to adhere to a constant activity protocol. Deviation from this line would be expected with respect to the intercept in any subject whose BMR represented a significantly different proportion of total expenditure compared with the others and with respect to the slope if this proportion altered throughout pregnancy. Subject no. 3 showed both of these features, and her data formed a separate regression $(r 0.99, P<0.001)$. At the start of pregnancy her BMR was $79 \cdot 2 \%$ of $24 \mathrm{~h}$ expenditure and at 36 weeks this had risen to $84.5 \%$. The decrease in the non-BMR component of daily expenditure in this subject as pregnancy progressed indicates energy-sparing reductions in the cost of physical activity and thermogenesis. It seems unlikely that these reductions were a consequence of familiarization with the calorimetry procedure since the changes were most pronounced in late pregnancy.

Fig. 8 shows total 24 h energy expenditure partitioned into the three components of basal metabolism, exercise and the residual representing the sum of all minor physical movements and diet-induced thermogenesis. Averaged over all subjects the net cost (after subtracting BMR) of the 120 min of exercise was only 9.6 (SD 0.9 ) \% of total expenditure. The net cost of minor movements and thermogenesis over the remaining 1320 min averaged only $14 \cdot 3$ (SD $2 \cdot 1$ ) $\%$ of 24 h expenditure. The within-subject mean (and 1 SD) calculated over 


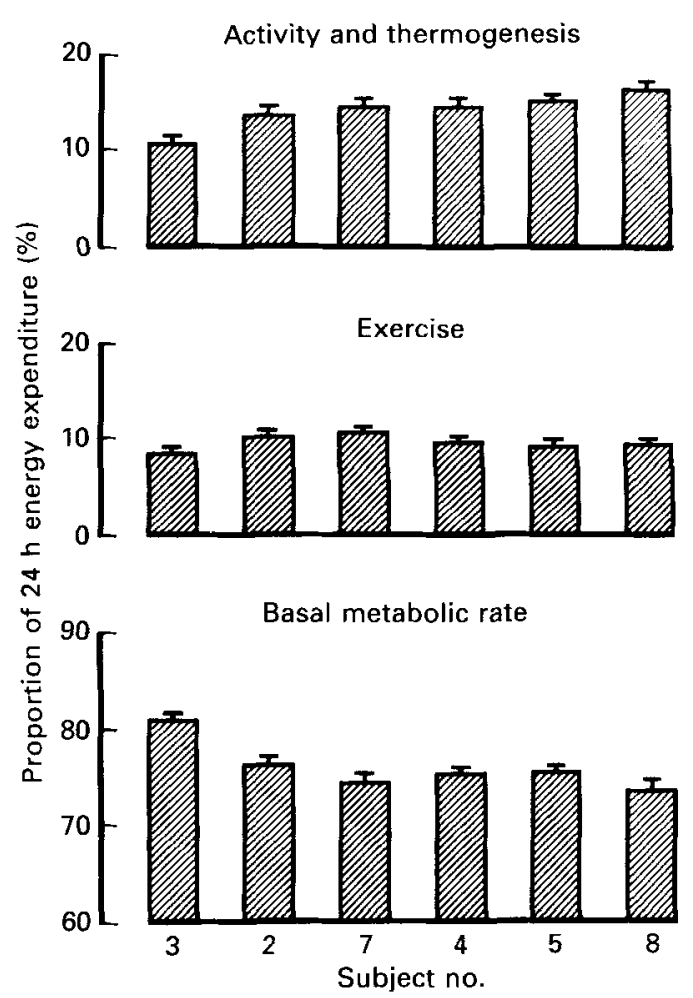

Fig. 8. Partition of $24 \mathrm{~h}$ energy expenditure into its major components for six healthy, well-nourished women. Values are within-subject averages (standard deviations are represented by vertical bars) calculated for all calorimeter measurements including non-pregnant, non-lactating. Cost of exercise was calculated as the gross cost of cycling, stepping, dressing and undressing periods.

all measurements including the NPNL baseline are shown in Fig. 8. The outstanding feature is the extremely small standard deviations in view of the fact that the measurements were made at 6-week intervals and spanned the whole of pregnancy. Analysis of variance demonstrated highly significant between-subject differences in the proportion of energy expended as BMR ( $F 17.0$, df 5,32, $P<0.001)$ as exercise $(F 11 \cdot 3$, df $5,32, P<0.001)$, and as minor physical movements and thermogenesis $(F 10.0, \mathrm{df} 5,32, P<0.001)$. Once again subject no. 3 differed from the others and expended considerably less energy on exercise, minor movements and activity. It is interesting to note that she was the most overweight subject with a body fat content of $40.8 \%$ before pregnancy.

Potential changes in the efficiency of muscular work during pregnancy were investigated using the standardized weight-dependent and weight-independent exercises. Figs. 9 and 10 show changes in the gross cost of the exercises expressed per kg body-weight. The average cost of cycling decreased to a plateau $16 \%$ below the NPNL value at 24 weeks gestation. The average gross cost of stepping also decreased when expressed per $\mathrm{kg}$ body-weight to reach a plateau $7 \%$ below the NPNL value. In contrast to the BMR values there was little evidence of significant between-subject variation in their response to pregnancy. When expressed on a whole-body basis the average gross cost of cycling was $96 \cdot 0,95 \cdot 4,96 \cdot 0,92 \cdot 7$, 97.8 and $101.0 \%$ of the NPNL value from 6 to 36 weeks. The gross cost of stepping was $98 \cdot 8,97.5,97 \cdot 7,100 \cdot 8,109 \cdot 0(P<0.01$, paired $t$ test $)$ and $113.2 \%(P<0.01)$ of the NPNL value. 

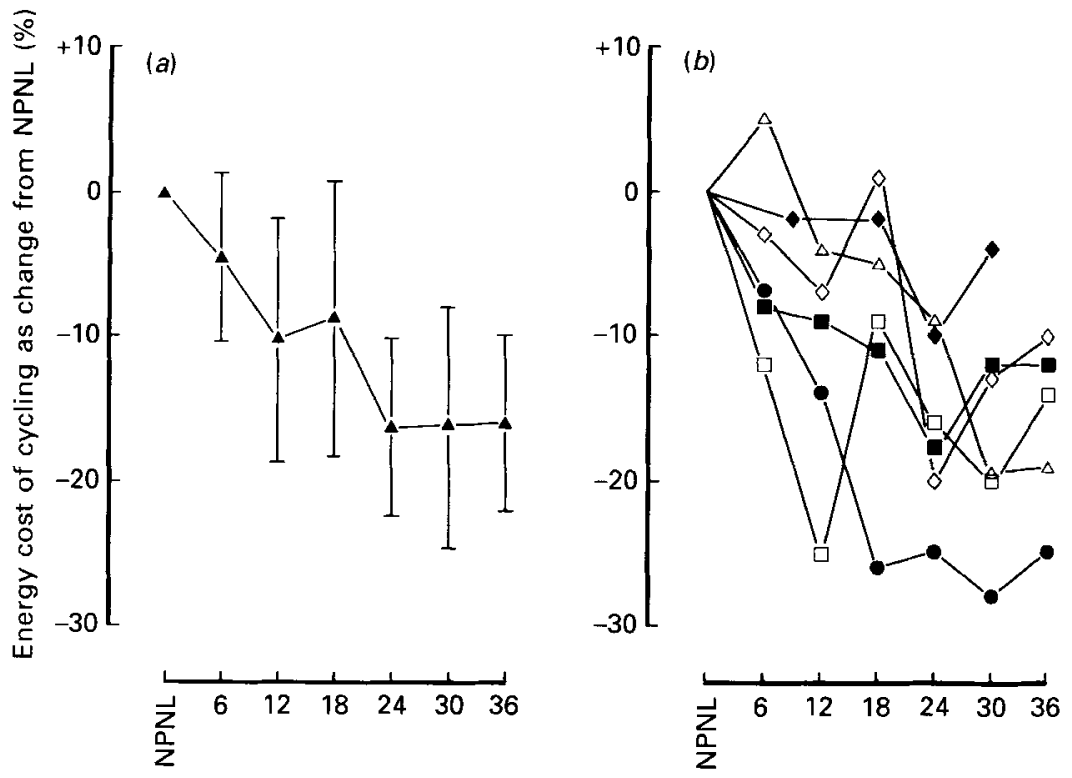

Stage of pregnancy (weeks)

Fig. 9. Changes in the gross energy cost of weight-independent exercise during pregnancy for six healthy, wellnourished women. Subject nos: $2(\bullet), 3(\square), 4(\square), 5(\triangle), 7(\diamond), 8(\diamond)$. Values represent the gross cost of fixedload bicycle ergometer exercise expressed per $\mathrm{kg}$ body-weight. (a) Group means and standard deviations represented by vertical bars; (b) individual values. Average non-pregnant, non-lactating (NPNL) expenditure was 200 (sD 11) J/kg per min.
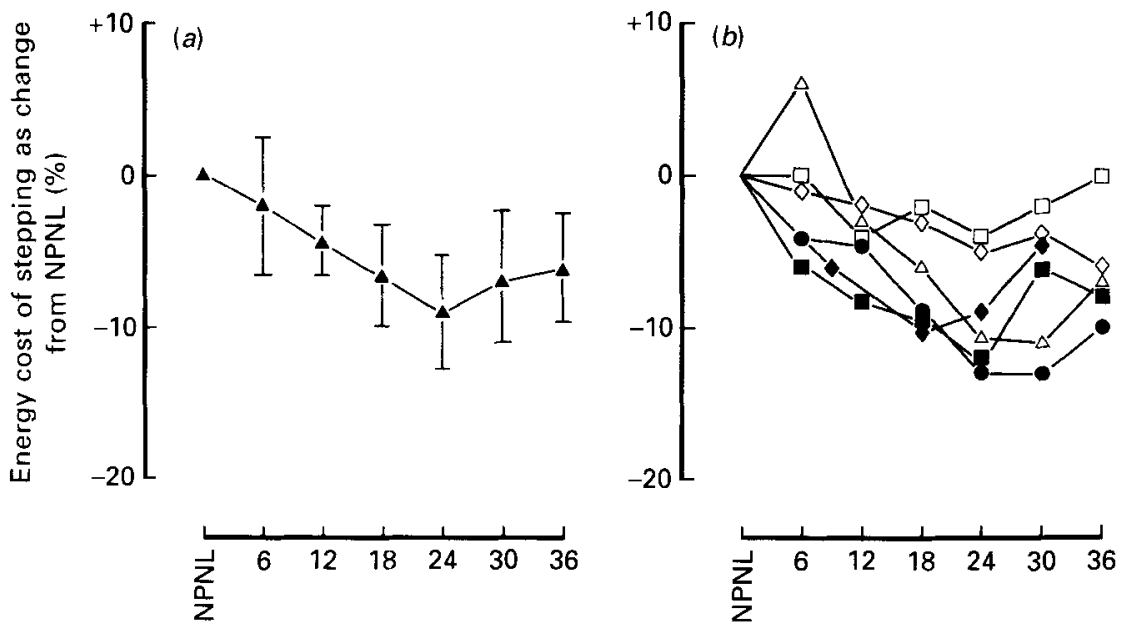

Stage of pregnancy (weeks)

Fig. 10. Changes in the gross energy cost of weight-dependent exercise during pregnancy for six healthy, wellnourished women. Subject nos: $2(\bullet), 3(\square), 4(\square), 5(\triangle), 7(\diamond), 8(\diamond)$. Values represent the gross cost of block stepping expressed per $\mathrm{kg}$ body-weight. (a) Group means and standard deviations represented by vertical bars; (b) individual values. Average non-pregnant, non-lactating (NPNL) expenditure was 226 (SD 20) J/kg per min. 


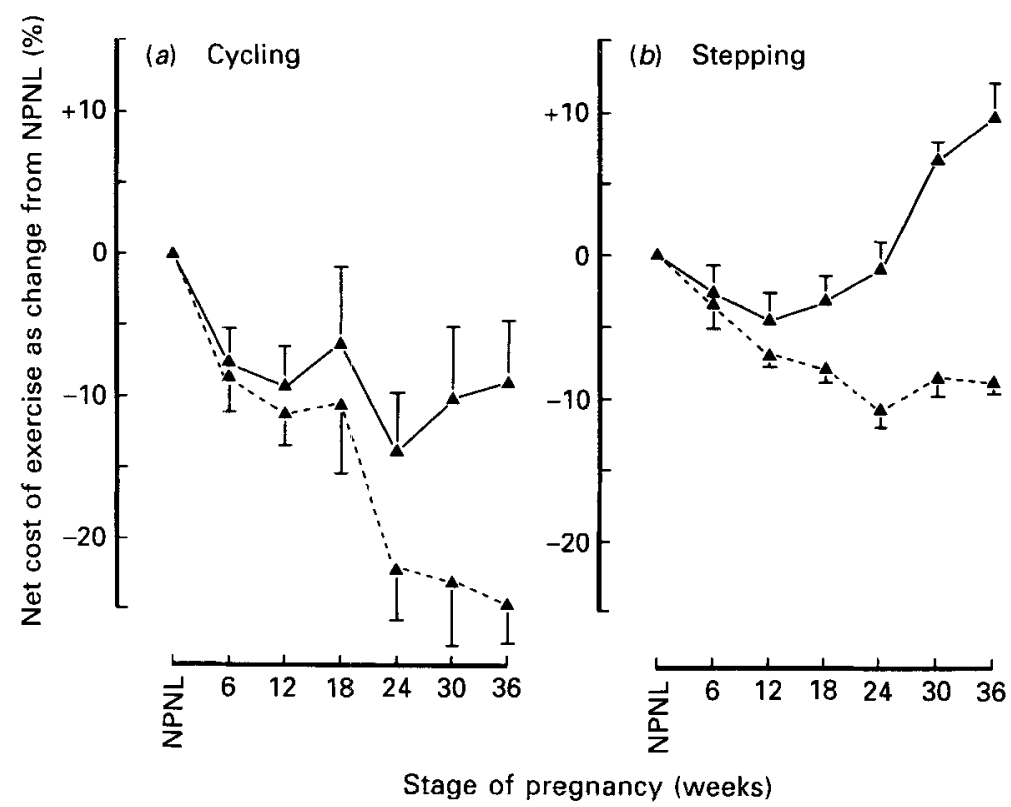

Fig. 11. Changes in the net cost of exercise during pregnancy for six heathy, well-nourished women. ( - ) Absolute cost; (--) cost per kg body-weight. Points are means with their standard errors represented by vertical bars. Net cost was calculated as gross cost minus basal metabolic rate. Average non-pregnant, non-lactating (NPNL) net cost: (a) cycling 8.7 (SE 0.6) $\mathrm{kJ} / \mathrm{min}$ or 134 (SE 9) $\mathrm{J} / \mathrm{kg}$ per min; (b) stepping 10.4 (SE 1.0) $\mathrm{kJ} / \mathrm{min}$ or 161 (SE 9) $\mathrm{J} / \mathrm{kg}$ per min.

Fig. 11 illustrates the group mean changes in the net cost of the two exercises calculated by subtracting BMR from the gross expenditure. The net cost of cycling decreased by about $10 \%$ when expressed on a whole-body basis and by $26 \%(P<0.01$ at $12,24,30$ and 36 weeks by paired $t$ test) expressed per $\mathrm{kg}$. The net cost of stepping decreased slightly and then increased to $+10 \%$ above NPNL when expressed on a whole-body basis $(P<0.02$ at 24-36 weeks), but decreased by $10 \%$ ( $P<0.001$ at $18-36$ weeks) when expressed per kg. The 'apparent' efficiencies of cycling and stepping increased from 18.5 (SD 0.6) and 13.6 (SD 0.4) \% in the NPNL state to maxima of 21.5 (SD 1.1) and 15.5 (SD 0.4) \% respectively. It should be noted that subtraction of BMR does not yield the true net cost of exercise from which true efficiencies of muscular work could be computed. This would necessitate subtracting the energy costs of cycling at zero work load and stepping on the spot respectively.

\section{DISCUSSION}

The initial hypothesis addressed by the present study was that pregnant women are capable of energy-sparing metabolic adaptations in basal metabolism, dietary thermogenesis or exercise. Such adaptations might explain two paradoxical observations. First, that many women in affluent societies show very small increments in food intake during pregnancy which do not match their theoretical extra energy requirements (Durnin et al. 1985; Durnin, 1987). Second, that women in many developing countries achieve a successful outcome to pregnancy in spite of being chronically undernourished (Prentice \& Whitehead, 1987). The results presented here show little scope for energy savings with respect to dietinduced thermogenesis or exercise, but considerable scope for adaptations in basal metabolism. 
The most important finding is that individual women showed very characteristic changes in basal metabolism, which followed consistent patterns within each subject, but were highly variable between subjects. At 36 weeks the percentage change in BMR above the NPNL baseline varied over a four-fold range from 8.6 to $35.4 \%$. The cumulative maintenance costs of pregnancy (computed as the area under each subject's BMR curve) had a CV of $93 \%$. Since basal metabolism constitutes such a large proportion of total energy expenditure this variability will have an important influence on daily energy requirements.

In view of the extent of this variability and its importance in determining overall energy needs it is surprising that it has not been noted earlier. However, many previous studies did not include longitudinal measurements and most have concentrated on defining the average increase in BMR within different groups of women. This is particularly true of several recent studies (Durnin, 1987). Under such circumstances, when the aim is to obtain an accurate estimate of group-mean changes in metabolism, individual variability becomes a positive disadvantage which has to be overcome by increasing the sample size. Several studies provide no quantitative evidence of variability since standard deviations have been omitted (Tuazon et al. 1987; van Raaij et al. 1987), but a crude estimate can be calculated from error bars illustrated in a number of other studies. At 36 weeks gestation the CV for the change in BMR relative to NPNL baseline values varies between 65 and $90 \%$ (Forsum et al. 1985; Durnin et al. 1987; Illingworth et al. 1987; Throngprasert et al. 1987). These values are actually larger than the $51 \%$ observed in this study, confirming that the present findings are not the result of chance observations in metabolically abnormal subjects.

An additional reason why the high level of inter-individual variability noted in the present study has not previously been commented on may be that the analytical techniques and experimental protocols employed in some studies have not been sufficiently precise to discriminate intra-individual differences from measurement noise. In the present study the test-retest CV (which includes both subject and instrumentation noise) was only $1.9 \%$ for BMR and $1.0 \%$ for $24 \mathrm{~h}$ energy expenditure. This high level of precision, together with the rigidly defined experimental protocol, which excluded most behavioural noise, revealed the very consistent within-subject trends illustrated in Fig. 3.

The differential changes in BMR were caused by mass-specific changes in metabolism rather than by differences in the amount of LBM gained by each subject. This finding may be important in the wider context of human energy balance. Currently there is considerable interest in the flexibility of metabolic rate and the question of whether such flexibility can accommodate changes in energy intake in an auto-regulatory manner (Sukhatme \& Margen, 1982). However, there is surprisingly little reliable information on within-subject differences in BMR over time. During the menstrual cycle BMR has been reported to fluctuate by 6-7\% (Solomon et al. 1982) although other studies have failed to demonstrate any significant fluctuation. A substantial mass-specific reduction in BMR (about 15\%) occurs in response to semi-starvation (Apfelbaum et al. 1971), but convincing evidence of metabolic flexibility has not been demonstrated in other circumstances once allowance has been made for confounding variables such as diet-induced thermogenesis. Indeed recent studies in healthy, free-living men on self-selected diets have demonstrated only a small degree of variability (Soares \& Shetty, 1987; A. M. Prentice, unpublished results). The observation in the present study of changes in BMR per $\mathrm{kg} \mathrm{LBM}$ over a $28 \%$ range from $-9 \cdot 2$ to $+18.6 \%$ relative to NPNL strongly suggests that pregnancy is a special case in which metabolic rate is particularly flexible.

Studies conducted in our Gambian field-station as part of a multi-centre collaborative project (Durnin, 1987) have suggested a link between maternal energy sufficiency and the metabolic response during pregnancy (Lawrence et al. 1987). The chronically under- 
nourished Gambian women showed an initial decrease in BMR during pregnancy followed by a much lower increase at term than occurs on average in well-nourished women. These changes were very similar to those shown by the energy-sparing subjects in the present study. Furthermore, the Gambian women were subdivided into two groups, one of which received an energy-dense dietary supplement during pregnancy (Lawrence et al. 1987). This group showed a significantly greater increase in BMR than the unsupplemented women suggesting that the energy cost of pregnancy varied in response to energy supply.

We have looked for supporting evidence for this hypothesis within the present data-set by attempting to identify correlations which might explain the between-subject heterogeneity in response to pregnancy. The dependent variable was defined as the percentage change in BMR relative to the NPNL value. Due to the high degree of ranking after 12 weeks gestation, similar correlations were obtained irrespective of which measurement was used. The prepregnant independent variables investigated were: body-weight, $\%$ body fat, BMR $/ \mathrm{kg}$ LBM and energy intake $/ \mathrm{kg}$ body-weight. The change in BMR at 24 weeks was significantly correlated with maternal fatness prior to pregnancy $(r 0.841, P<0.005)$; thin mothers tended to be energy-sparing. If maternal fatness acts as a proxy variable for energy sufficiency this finding would support the hypothesis that metabolic adaptations counteract the effects of energy insufficiency or excess.

The range in the total cumulative costs of maintaining the products of conception (recorded as -16 to $+276 \mathrm{MJ}$ in the present study) cannot be compared with other studies since they all quote a single value derived as the area under the average BMR curve for the entire data-set. However, comparison of these group-mean values for the costs of maintaining gestation also suggests the presence of an interaction between energy intake and the metabolic response to pregnancy of the type noted in this study. The longitudinal data available from affluent Western women record maintenance costs of $123 \mathrm{MJ}$, present study; $126 \mathrm{MJ}$, Scotland (Durnin et al. 1987); 144 MJ, The Netherlands (van Raaij et al. 1987 ) and 111 MJ (up to 36 weeks), Sweden (Forsum et al. 1985). Expression of the values as multiples of the NPNL BMR, in order to normalize for differences in initial metabolic body size, gives values of $20 \cdot 1,22.5,22.5$ and $19 \cdot 8 \times$ NPNL BMR respectively. Thus, there is good agreement between average values from well-nourished women.

The values from Thai (Throngprasert et al. 1987) and Philippino (Tuazon et al. 1987) women, who were also reported to be adequately nourished, revealed energy costs of 100 and $79 \mathrm{MJ}$, or 18.9 and $15.8 \times$ NPNL BMR. Since these were estimated only over the last 27 weeks of gestation they appear to be compatible with the values from European women. In contrast, the energy-sparing adaptive patterns noted in the Gambian subjects produced mean maintenance costs for pregnancy of $-45 \mathrm{MJ}$ in unsupplemented women, or $+4 \mathrm{MJ}$ in the supplemented subgroup (Lawrence et al. 1987). Therefore both the within-group regression analysis reported in the present paper and the between-group analysis based on maternal energy supply suggest that maintaining gestation has a variable metabolic cost which is dependent on the mother's nutritional status.

Since basal metabolism constitutes $70-80 \%$ of total daily expenditure in sedentary women (Prentice et al. 1985) the potential for energy-sparing adaptations in diet-induced thermogenesis or exercise are limited. Illingworth et al. (1987) reported a significant reduction in dietary thermogenesis during mid-pregnancy in response to a standardized test meal. However, the reported difference amounted to only $0.6 \%$ of the energy ingested. In the present study we measured the sum of all diet-induced thermogenesis and minor physical movements over $24 \mathrm{~h}$. The mean value was $14.3 \%$ of total expenditure and this percentage remained very constant throughout pregnancy in each subject (average withinsubject $\mathrm{CV}$ was $6 \%$, which is equivalent to $0.8 \%$ of total expenditure). The constancy of these values indicates either that any changes in the cost of diet-induced thermogenesis were 
very small, or that they had been offset by equal and opposite changes in the energy expended on minor physical movements.

Although little energy is expended as gross physical activity in modern affluent subjects, it may constitute up to $50 \%$ of total expenditure at times of peak agricultural activity in the developing world (Prentice \& Whitehead, 1987). Potential changes in work efficiency may be significant under such circumstances. A review of the literature on changes in the energy cost of exercise during pregnancy reveals considerable disagreement between studies (Lotgering et al. 1985). This almost certainly arises from the fact that most studies have made cross-sectional comparisons of pregnant and non-pregnant women. The longitudinal measurements in the present study demonstrated that the absolute cost of weightindependent exercise remained unaltered throughout pregnancy, and that the cost of weight-dependent exercise increased but by less than the increase in body-weight. When either the gross or net costs of each exercise were expressed per $\mathrm{kg}$ body-weight, there were highly significant reductions in energy expenditure. It is unlikely that these changes represent a familiarization effect since the exercises were 6 weeks apart and since the increase in efficiency was progressive throughout pregnancy. It is possible that they resulted from a real training effect in pregnancy caused by the increased maternal body-weight. The recent multi-centre collaborative study in pregnancy has reported similar results based on a standardized treadmill exercise (Durnin, 1987).

The adaptive responses to pregnancy shown in the present study have a number of practical consequences. First, they make it difficult to offer prescriptive recommendations to individual pregnant women about desirable increments in energy intake, since prospectively it would be impossible to predict how their metabolism will respond. Second, they may represent a powerful selection trait, since neonatal mortality is closely correlated to infant birth weight, and since a mother's ability to adopt energy-sparing mechanisms may help to prevent intra-uterine growth retardation when dietary energy is scarce.

The authors thank the subjects for participating in this study, Dr M. Elia and Mrs $\mathbf{H}$. Tulett for providing clinical cover and Mrs E. Collard for preparing the calorimetery diets.

\section{REFERENCES}

Apfelbaum, M., Bostsarron, J. \& Lacatis, D. (1971). Effect of caloric restriction and excessive caloric intake on energy expenditure. American Journal of Clinical Nutrition 24, 1404-1409.

Brown, D., Cole, T. J., Dauncey, M. J., Marrs, R. W. \& Murgatroyd, P. R. (1984). Analysis of gaseous exchange in open-circuit indirect calorimetry. Medical and Biological Engineering and Computing 22, 333-338.

Dallosso, H. M., Murgatroyd, P. R. \& James, W. P. T. (1982). Feeding frequency and energy balance in adult males. Human Nutrition: Clinical Nutrition 36C, 25-39.

Durnin, J. V. G. A. (1987). Energy requirements of pregnancy. An integrated study in five countries: background and methods. Lancet ii, 895-896.

Durnin, J. V. G. A., McKillop, F. M., Grant, S. \& Fitzgerald, G. (1985). Is nutritional status endangered by virtually no extra intake during pregnancy? Lancet ii, 823-825.

Durnin, J. V. G. A., McKillop, F. M., Grant, S. \& Fitzgerald, G. (1987). Energy requirements of pregnancy in Scotland. Lancet ii, 897-900.

Forsum, E., Sadurskis, A. \& Wager, J. (1985). Energy maintenance cost during pregnancy in healthy Swedish women. Lancet i, 107-108.

Illingworth, P. J., Jung, R. T., Howie, P. W. \& Isles, T. E. (1987). Reduction in postprandial energy expenditure during pregnancy. British Medical Journal 294, 1573-1576.

Lawrence, M., Lawrence, F., Coward, W. A., Cole, T. J. \& Whitehead, R. G. (1987). Energy requirements of pregnancy in The Gambia. Lancet ii, 1072-1076.

Lotgering, F. K., Gilbert, R. D. \& Longo, L. D. (1985). Maternal and fetal responses to exercise during pregnancy. Physiological Reviews 65, 1-36.

Prentice, A. M., Coward, W. A., Davies, H. L., Murgatroyd, P. R., Goldberg, G. R., Black, A. E., Sawyer, M., Ashford, J. \& Whitehead, R. G. (1985). Unexpectedly low levels of energy expenditure in healthy women. Lancet i, 1419-1422. 
Prentice, A. M. \& Whitehead, R. G. (1987). The energetics of human reproduction. Zoological Society of London Symposia 57, 275-304.

Seitchik, J. (1967). Total body water and body density of pregnant women. Obstetrics and Gynecology 29, $155-166$.

Soares, M. J. \& Shetty, P. S. (1987). Long-term stability of metabolic rates in young adult males. Human Nutrition: Clinical Nutrition 41C, 287-290.

Solomon, S. J., Kurzer, M. S. \& Calloway, D. H. (1982). Menstrual cycle and basal metabolic rate in women. American Journal of Clinical Nutrition 36, 611-616.

Sukhatme, P. V. \& Margen, S. (1982). Autoregulatory homoeostatic nature of energy balance. American Journal of Clinical Nutrition 35, 355-365.

Thongprasert, K., Tanphaichitre, V., Valyasevi, A., Kittigool, J. \& Durnin, J. V G. A. (1987). Energy requirements of pregnancy in rural Thailand. Lancet ii, 1010-1012.

Tuazon, M. A. G., van Raaij, J. M. A., Hautvast, J. G. A. J. \& Barba, C. V. C. (1987). Energy requirements of pregnancy in the Philippines. Lancet ii, 1129-1131.

van Raaij, J. M. A., Vermaat-Miedema, S. H., Schonk, C. M., Peek, M. E. M. \& Hautvast, J. G. A. J. (1987). Energy requirements of pregnancy in The Netherlands, Lancet ii, 953-955.

Weir, J. B. deV. (1949). New methods for calculating metabolic rate with special reference to protein metabolism. Journal of Physiology 109, $1-9$. 